\title{
ON A QUATERNIONIC PICARD THEOREM
}

\author{
CINZIA BISI AND JÖRG WINKELMANN \\ (Communicated by Filippo Bracci)
}

\begin{abstract}
The classical theorem of Picard states that a non-constant holomorphic function $f: \mathbb{C} \rightarrow \mathbb{C}$ can avoid at most one value.

We investigate how many values a non-constant slice regular function of a quaternionic variable $f: \mathbb{H} \rightarrow \mathbb{H}$ may avoid.
\end{abstract}

\section{INTRODUCTION}

A function $f: \mathbb{C} \rightarrow \mathbb{C}$ which is given by a globally convergent power series $f(z)=\sum_{k=0}^{\infty} a_{k} z^{k}\left(a_{k} \in \mathbb{C}\right)$ is called an entire function. By the theorem of Picard, a non-constant entire function $f: \mathbb{C} \rightarrow \mathbb{C}$ can avoid at most one value [10, [1], [12].

Our goal is a similar statement for entire slice regular functions, i.e., for functions $f: \mathbb{H} \rightarrow \mathbb{H}$ (where $\mathbb{H}$ denotes the skew field of quaternions) which are given as a globally convergent power series $f(q)=\sum_{k=0}^{\infty} q^{k} a_{k}\left(a_{k} \in \mathbb{H}\right)$.

For a function $f: \mathbb{H} \rightarrow \mathbb{H}$ being "slice regular" is equivalent to the assumption that for every imaginary unit $I \in \mathbb{S}$ its restriction to $\mathbb{C}_{I}=\{x+y I: x, y \in \mathbb{R}\}$ is holomorphic with respect to the complex structures induced by left multiplication by $I$; see $[4,5]$.

Here we show the following:

(i) For every 2-dimensional real affine subspace $P$ of $\mathbb{H} \simeq \mathbb{R}^{4}$, there exists an entire slice regular function $f: \mathbb{H} \rightarrow \mathbb{H}$ such that $f(\mathbb{H})=\mathbb{H} \backslash P$. In particular, for every triple $q_{1}, q_{2}, q_{3} \in \mathbb{H}$ there is an entire slice regular function avoiding these three values.

(ii) Let $q_{1}, \ldots, q_{5} \in \mathbb{H}$ be in general position (i.e., these five quaternions are not contained in any 3 -dimensional real affine subspace of $\mathbb{H}$ ). Then every entire slice regular function avoiding all these five values must be constant. In particular, for every non-constant entire slice regular function the image is dense in $\mathbb{H}$.

We do not know whether an entire slice regular function may avoid a generic choice of four quaternionic numbers.

A key tool is the following fundamental correspondence (see Proposition 2.2):

Let $f$ be a slice regular function and let $F$ be its stem function. Let $x, y \in \mathbb{R}$ and $c \in \mathbb{H}$. Then there exists an imaginary unit $I \in \mathbb{S}$ such that $f(x+y I)=c$ if and only if $F(x+y i)-c \otimes 1$ is a zero divisor in the algebra $\mathbb{H} \otimes \mathbb{C}$.

Received by the editors September 30, 2019, and, in revised form, April 7, 2020, June 13, 2020, and June 24, 2020.

2010 Mathematics Subject Classification. Primary 30G35.

The two authors were partially supported by GNSAGA of INdAM. The first author was also partially supported by PRIN Varietá reali e complesse: geometria, topologia e analisi armonica. 
Maybe this work can be of some inspiration in studying hyperbolic quaternionic slice regular manifolds. Indeed recently many examples of quaternionic slice regular manifolds have been introduced; see for example [2], [1].

\section{Preparations}

2.1. Quaternions. The quaternionic numbers are a real 4-dimensional skew field $\mathbb{H}$, which may be described as the non-commutative $\mathbb{R}$-algebra with 1 , generated by $I, J, K$ with $I^{2}=J^{2}=K^{2}=-1, K=I J=-J I, I=J K=-K J$ and $J=K I=-I K$.

The set of all elements $q \in \mathbb{H}$ with $q^{2}=-1$ is called the set of imaginary units and denoted by $\mathbb{S}$.

One may check easily that

$$
\mathbb{S}=\left\{c_{2} I+c_{3} J+c_{4} K: c_{i} \in \mathbb{R}, \sum_{i=2}^{4} c_{i}^{2}=1\right\} .
$$

2.2. Slice regular functions and stem functions. We recall the theory of slice regular functions and their stem functions $([5,6]$ ).

An entire slice regular function $f: \mathbb{H} \rightarrow \mathbb{H}$ is a function which is given by a globally convergent power series $f(q)=\sum_{k=0}^{\infty} q^{k} a_{k}$ (with $a_{k} \in \mathbb{H}$ ).

A stem function $F$ is a holomorphic map from $\mathbb{C}$ to the $\mathbb{C}$-algebra $\mathbb{H}_{\mathbb{C}}=\mathbb{H} \otimes_{\mathbb{R}} \mathbb{C}$ such that $\overline{F(z)}=F(\bar{z})$. The tensor product $\mathbb{H} \otimes_{\mathbb{R}} \mathbb{C}$ inherits a complex structure from its second factor, $\mathbb{C}$, hence it makes sense to talk about holomorphicity and complex conjugation.

In explicit terms, the stem function $F$ associated to a slice regular function $f(q)=\sum_{k=0}^{\infty} q^{k} a_{k}$ may be defined as $F(z)=\sum_{k=0}^{\infty} a_{k} \otimes z^{k}$.

Equivalently, the correspondence may be described as follows:

$$
F(x+y i)=F_{1}(x+y i) \otimes 1+F_{2}(x+y i) \otimes i
$$

with

$$
F_{1}(x+y i)=\frac{1}{2}(f(x+y I)+f(x-y I))
$$

and

$$
F_{2}(x+y i)=-\frac{1}{2} I(f(x+y I)-f(x-y I)) .
$$

For a slice regular function $f$ the terms on the right hand side can be shown to be independent of the choice of the imaginary unit $I$.

Conversely, one has

$$
f(x+y H)=F_{1}(x+y i)+H F_{2}(x+y i) \quad \forall x, y \in \mathbb{R}, H \in \mathbb{S} .
$$

2.3. A remarkable quadric in $\mathbb{H}_{\mathbb{C}}$. The euclidean scalar product on $\mathbb{H} \simeq \mathbb{R}^{4}$ induces a complex symmetric bilinear form $\langle$,$\rangle on \mathbb{H}_{\mathbb{C}}$. Explicitly: $\langle z, w\rangle=$ $\sum_{i=1}^{4} z_{i} w_{i}$.

We observe that $\mathbb{H}_{\mathbb{C}}$ naturally carries the structure of an $\mathbb{R}$-algebra.

Both the field of complex numbers $\mathbb{C}$ and the quaternionic skew field $\mathbb{H}$ embed into $\mathbb{H}_{\mathbb{C}}$ via $z \mapsto 1 \otimes z$, resp., $q \mapsto q \otimes 1$. In this way we may regard $\mathbb{C}$ and $\mathbb{H}$ as subrings of $\mathbb{H}_{\mathbb{C}}$. 
Proposition 2.1. Let $v=1 \otimes v_{0}+I \otimes v_{1}+J \otimes v_{2}+K \otimes v_{3}=v^{\prime} \otimes 1+v^{\prime \prime} \otimes i$ (with $\left.v_{i} \in \mathbb{C}, v^{\prime}, v^{\prime \prime} \in \mathbb{H}\right)$ be an element of $\mathbb{H}_{\mathbb{C}}$.

Then the following are equivalent:

(i) $v$ is a zero divisor, i.e., there exists an element $w \in \mathbb{H}_{\mathbb{C}}, w \neq 0$ with $w \cdot v=0$.

(ii) $\langle v, v\rangle=0$, i.e., $\sum_{i=0}^{3} v_{i}^{2}=0$.

(iii) There exists an imaginary unit $H \in \mathbb{S}$ such that $H v^{\prime}=v^{\prime \prime}$. (Geometrically: The vectors $v^{\prime}$ and $v^{\prime \prime}$ are orthogonal.)

The above equivalence (i) $\Longleftrightarrow$ (ii) is contained in [13] where it is attributed to Hamilton, while (ii) $\Longleftrightarrow$ (iii) may be deduced from the work of Mongodi ([7]). In addition, these equivalences may be obtained as a special case of a result of Ghiloni and Perotti ([6, Theorem 17 on page 1679]).

For the convenience of the reader we nevertheless give a proof here.

Proof. (i) $\Longrightarrow$ (iii): We assume that $v$ is a zero divisor (but $v \neq 0$ ). Since $\mathbb{H}$ has no zero divisors, it follows that $v^{\prime}, v^{\prime \prime} \neq 0$. Now $v^{\prime} \in \mathbb{H}^{*}$ and $v$ being a zero divisor, imply that $v \cdot\left(\left(v^{\prime}\right)^{-1} \otimes 1\right)$ is again a zero divisor. Hence we may assume that $v^{\prime}=1$. The same reasoning also shows that we can find an element $w=w^{\prime}+w^{\prime \prime} \otimes i$ with $w^{\prime}=1$ and $w \cdot v=0$. Thus we obtain

$$
0=w \cdot v=\left(1+w^{\prime \prime} \otimes i\right) \cdot\left(1+v^{\prime \prime} \otimes i\right)=\left(1-w^{\prime \prime} v^{\prime \prime}\right) \otimes 1+\left(v^{\prime \prime}+w^{\prime \prime}\right) \otimes i .
$$

Hence $v^{\prime \prime}=-w^{\prime \prime}$ and $\left(v^{\prime \prime}\right)^{2}=-v^{\prime \prime} w^{\prime \prime}=-1$, i.e., $v^{\prime \prime} \in \mathbb{S}$. In particular, $v^{\prime \prime}=H \cdot 1=$ $H \cdot v^{\prime}$ for some $H \in \mathbb{S}$.

(iii) $\Longrightarrow$ (i): We have $v=(1 \otimes 1+H \otimes i) \cdot v^{\prime}$. Define $w=1 \otimes 1-H \otimes i$. Then $w \cdot v=0$, as easily seen by explicit calculation.

(iii) $\Longleftrightarrow$ (ii): Note that

$$
\langle v, v\rangle=\left\langle v^{\prime}+v^{\prime \prime} \otimes i, v^{\prime}+v^{\prime \prime} \otimes i\right\rangle=\left\langle v^{\prime}, v^{\prime}\right\rangle-\left\langle v^{\prime \prime}, v^{\prime \prime}\right\rangle+2 i\left\langle v^{\prime}, v^{\prime \prime}\right\rangle .
$$

Hence $\langle v, v\rangle=0$ iff $v^{\prime}$ and $v^{\prime \prime}$ have the same norm and are orthogonal to each other. This in turn is equivalent to the existence of an imaginary unit $H \in \mathbb{S}$ with $v^{\prime \prime}=H v^{\prime}$.

Thus the set of all zero divisors of $\mathbb{H}_{\mathbb{C}}$ is a quadric subvariety of $\mathbb{H}_{\mathbb{C}} \simeq \mathbb{C}^{4}$. This quadric has also been investigated by Mongodi ([7]), who pointed out the relevance for the zero locus, but not the relation with zero divisors of the algebra $\mathbb{H}_{\mathbb{C}}$.

2.4. Zeros. Let $f$ be a slice function and let $F$ denote its stem function. Write $F=F_{1} \otimes 1+F_{2} \otimes i$, with $F_{h}: \mathbb{C} \rightarrow \mathbb{H}$. Since

$$
f(x+y I)=F_{1}(x+y i)+I F_{2}(x+y i) \quad \forall x, y \in \mathbb{R}, I \in \mathbb{S},
$$

this implies

$$
f(x+y I)=0 \Longleftrightarrow F_{1}(x+y i)=-I F_{2}(x+y i),
$$

The following result is implied by Proposition 2.1. but may also be deduced from [7. Proposition 4.1] in combination with Corollary 3.4 of [7]:

Proposition 2.2. Let $f: \mathbb{H} \rightarrow \mathbb{H}$ be a slice regular function and let $F: \mathbb{C} \rightarrow$ $\mathbb{H}_{\mathbb{C}}=\mathbb{H} \otimes_{\mathbb{R}} \mathbb{C}$ be its stem function. Let $x, y \in \mathbb{R}$. Then the following conditions are equivalent:

(i) There exists an imaginary unit $H \in \mathbb{S}$ with $f(x+y H)=0$.

(ii) $\langle F(x+y i), F(x+y i)\rangle=0$.

(iii) $F(x+y i)$ is a zero divisor in the algebra $\mathbb{H} \otimes_{\mathbb{R}} \mathbb{C}$. 
This has the following consequence: let $c \in \mathbb{H}$. Then a slice regular function $f$ avoids $c$ as value (i.e., $f(\mathbb{H}) \subset \mathbb{H} \backslash\{c\}$ ) if and only if $z \mapsto F(z)-c \otimes 1$ has no zero which happens if and only if the entire function

$$
Q_{c}: z \mapsto\langle F(z)-c, F(z)-c\rangle=\langle F(z), F(z)\rangle-2\langle F(z), c\rangle+\langle c, c\rangle
$$

has no zeros.

\section{Avoiding FIVE GENERIC VAlues}

The purpose of this section is to show that a non-constant entire slice regular function cannot avoid five values if these are generic in the following sense: there is no real 3-dimensional affine subspace of $\mathbb{H} \simeq \mathbb{R}^{4}$ containing all of them.

We start with some preparations.

First we recall two results of Noguchi on holomorphic curves in semi-abelian varieties. Here we do not need to deal with arbitrary semi-abelian varieties, it suffices to know that $\left(\mathbb{C}^{*}\right)^{g}$ is a semi-abelian variety.

Proposition 3.1 (Logarithmic Bloch Ochiai theorem). Let $f: \mathbb{C} \rightarrow G=\left(\mathbb{C}^{*}\right)^{g}$ be a holomorphic map and let $X$ denote the Zariski closure of its image.

Then $X$ is an orbit of an algebraic subgroup $H$ of $G=\left(\mathbb{C}^{*}\right)^{g}$ (acting by left multiplication $)$, i.e., there is an element $\lambda=\left(\lambda_{1}, \ldots, \lambda_{g}\right) \in G=\left(\mathbb{C}^{*}\right)^{g}$ such that

$$
X=\{\lambda \cdot h: h \in H\} .
$$

See Main Theorem (i) in [8].

Proposition 3.2. Let

$$
f: \Delta^{*}=\{z \in \mathbb{C}: 0<|z|<1\} \rightarrow G=\left(\mathbb{C}^{*}\right)^{g} \subset \bar{G}=\left(\mathbb{P}_{1}\right)^{g}
$$

be a holomorphic map and let $X$ denote the Zariski closure of its image. Define

$$
\operatorname{Stab}(X)=\{g \in G: g \cdot x \in X \forall x \in X\} .
$$

If $\operatorname{Stab}(X)$ is discrete, then $f$ extends to a holomorphic map from $\Delta$ to $\bar{G}$.

Proof. This is a consequence of Theorem 4.5. of 9], applied with taking the Zariski closure of $f\left(\Delta^{*}\right)$ as $X$. In the notation of [9] non-extendibility of $f$ implies $f\left(\Delta^{*}\right) \subset$ $W$. Since we take $X$ to be the Zariski closure of the image of $f$, the inclusion $f\left(\Delta^{*}\right) \subset W$ implies $X=W$. In view of Lemma 4.1 in [9] the condition $X=W$ implies that $\operatorname{Stab}(X)$ is not discrete.

Proposition 3.3. Let $Z$ be an algebraic subvariety of $G=\left(\mathbb{C}^{*}\right)^{5}$. Assume that there exists a non-constant holomorphic map $g: \mathbb{C} \rightarrow Z$ with $g(z)=\overline{g(\bar{z})}$ for all $z \in \mathbb{C}$.

Then there exist $\alpha_{1}, \ldots, \alpha_{5} \in \mathbb{R}^{*}$ and $\left(m_{1}, \ldots, m_{5}\right) \in \mathbb{Z}^{5} \backslash\{(0, \ldots, 0)\}$ such that $\zeta\left(\mathbb{C}^{*}\right) \subset Z$ for

$$
\zeta(z) \stackrel{\text { def }}{=}\left(\alpha_{1} z^{m_{1}}, \ldots, \alpha_{5} z^{m_{5}}\right) .
$$

Proof. The Zariski closure of the image $g(\mathbb{C})$ in $G$ is an orbit of an algebraic subgroup $H$ of $G$ acting by multiplication (Proposition 3.1). We choose a connected 1-dimensional algebraic subgroup $T$ of $H$. Such a subgroup $T$ is isomorphic to $\mathbb{C}^{*}$ and parametrized by a map $\zeta_{0}: \mathbb{C}^{*} \rightarrow G=\left(\mathbb{C}^{*}\right)^{5}$ given as

$$
\zeta_{0}(z) \stackrel{\text { def }}{=}\left(z^{m_{1}}, \ldots, z^{m_{5}}\right) .
$$


Define $\alpha=\left(\alpha_{1}, \ldots, \alpha_{5}\right) \stackrel{\text { def }}{=} g(0)$. The condition $g(z)=\overline{g(\bar{z})}$ implies that $\alpha_{i} \in \mathbb{R}$ for all $i \in\{1, \ldots, 5\}$. By our construction the $H$-orbit through $\alpha$ must be contained in $Z$. It follows that $\zeta\left(\mathbb{C}^{*}\right) \subset Z$ for

$$
\zeta(z)=\zeta_{0}(z) \cdot \alpha=\left(\alpha_{1} z^{m_{1}}, \ldots, \alpha_{5} z^{m_{5}}\right) .
$$

Proposition 3.4. Let $c_{1}, \ldots, c_{4}$ be a basis of the real vector space $\mathbb{H}$. Let $M \in$ $\operatorname{Mat}(4 \times 4, \mathbb{R})$ be a positive definite symmetric real matrix. Let $Z$ denote the zero set of the function $\psi$ in $G=\left(\mathbb{C}^{*}\right)^{5}$ where

$$
\psi\left(v_{1}, \ldots, v_{4} ; p\right)=p-w^{t} M w, \quad\left(v=\left(v_{1}, \cdots, v_{4}\right) \in \mathbb{C}^{4}, p \in \mathbb{C}\right)
$$

with

$$
w=v-\left(\begin{array}{c}
p+\left\langle c_{1}, c_{1}\right\rangle \\
\vdots \\
p+\left\langle c_{4}, c_{4}\right\rangle
\end{array}\right) .
$$

Let $\alpha_{i} \in \mathbb{R}^{*}$ and $m_{i} \in \mathbb{Z}$ such that the image of the map $\zeta: \mathbb{C}^{*} \rightarrow G$ given as

$$
\zeta(z) \stackrel{\text { def }}{=}\left(\alpha_{1} z^{m_{1}}, \ldots, \alpha_{5} z^{m_{5}}\right)
$$

is contained in $Z$ (i.e., $\left.\zeta\left(\mathbb{C}^{*}\right) \subset Z\right)$.

Then $m_{i}=0$ for all $i \in\{1, \ldots, 5\}$, i.e., $\zeta$ must be constant.

Proof. We discuss the coefficients of the Laurent series $\sum_{k \in \mathbb{Z}} b_{k} z^{k}$ of the holomorphic function $z \mapsto(\psi \circ \zeta)(z)$ defined on $\mathbb{C}^{*}$. Since $\psi \circ \zeta \equiv 0$ due to $\zeta\left(\mathbb{C}^{*}\right) \subset Z$, we know that $b_{k}=0$ for all $k \in \mathbb{Z}$. On the other hand, the Laurent coefficients $b_{k}$ depend on the matrix $M$ and the coefficients $\alpha_{i}, m_{i}$. Using these facts we will see that we arrive at a contradiction if we assume that $\zeta$ is not constant.

We start by observing that $\psi$ is a polynomial map of degree 2 whose purely quadratic term is given by

$$
\psi_{2}(v ; p)=-(v-p d)^{t} M(v-p d) \quad \text { with } d=(1, \ldots, 1)^{t} .
$$

We may replace $\zeta$ with its composition with the inverse element map $z \mapsto 1 / z$ and thereby assume $m_{5} \geq 0$. By permuting variables we may also assume that

$$
m_{1} \leq m_{2} \leq m_{3} \leq m_{4} .
$$

Let us now assume that $\zeta$ is not constant, i.e., let us assume that $\left(m_{1}, \ldots, m_{5}\right) \neq$ $(0, \ldots, 0)$. Our strategy is to show that the Laurent series of $\psi \circ \zeta$ cannot vanish unless $\left(m_{1}, \ldots, m_{5}\right)=(0, \ldots, 0)$.

Case 1. We assume $m_{1}<0$.

Fix $k$ such that $m_{i}=m_{1}$ for $1 \leq i \leq k$ and $m_{i}>m_{1}$ for $k<i \leq 4$. We consider the Laurent coefficient of degree $2 m_{1}$. Note that $\zeta$ has no homogeneous component of degree less that $m_{1}$. Recall that $\psi$ is a quadratic polynomial. It follows that $\psi \circ \zeta$ has no homogeneous component of degree less that $2 m_{1}$ and that the homogeneous component of degree $2 m_{1}$ equals $\left(\psi_{2} \circ \zeta\right)_{2 m_{1}}$ where $\psi_{2}$ is the purely quadratic part of $\psi$ and $\left(\psi_{2} \circ \zeta\right)_{2 m_{1}}$ is the homogeneous component of $\psi_{2} \circ \zeta$ of degree $2 m_{1}$. Thus $\left(\psi_{2} \circ \zeta\right)_{2 m_{1}}=b_{2 m_{1}} z^{2 m_{1}}$.

By the definition of $\psi$ and $\zeta$, it follows that $b_{2 m_{1}}=-u^{t} M u$ with

$$
u=\left(\alpha_{1}, \ldots, \alpha_{k}, 0, \ldots, 0\right) .
$$


But $M$ is positive definite and the $\alpha_{i}$ are all real and non-zero. Hence $u^{t} M u>0$, contradicting $\psi \circ \zeta \equiv 0$.

Case 2. We assume $m_{5}>0$ and $m_{1} \geq 0$.

Fix $k \in\{1, \ldots, 4\}$ such that $m_{i}=0$ iff $i \leq k$. Here we investigate the constant term of the Laurent series of $\psi \circ \zeta$, i.e., its degree-0-coefficient.

This is $b_{0}=-u^{t} M u$ with

$$
u=\left(\alpha_{1}+\left\langle c_{1}, c_{1}\right\rangle, \ldots, \alpha_{k}+\left\langle c_{k}, c_{k}\right\rangle,\left\langle c_{k+1}, c_{k+1}\right\rangle, \ldots,\left\langle c_{4}, c_{4}\right\rangle\right) .
$$

We employ again the facts that $M$ is positive definite and $u$ is real. Hence $u^{t} M u=0$ requires that $u$ is the zero vector. Because $\left\langle c_{i}, c_{i}\right\rangle>0$, it follows that $k=4$. Thus $m_{i}=0$ for all $i<5$. But now it follows that the degree $2 m_{5}$-term is $-v^{t} M v$ with

$$
v=\left(\alpha_{5}, \ldots, \alpha_{5}\right)
$$

which yields a contradiction.

Case 3. We assume $m_{5}=0$ and $m_{1} \geq 0$.

Then $m_{4}=\max \left\{m_{1}, \ldots, m_{5}\right\}$ and we discuss the term of degree $2 m_{4}$. Let $k$ be such that $m_{i}=m_{4}$ iff $4 \geq i \geq k$. Then the degree $2 m_{4}$-coefficient of the Laurent series equals $-u^{t} M u$ with

$$
u=\left(0, \ldots, \alpha_{k}, \ldots, \alpha_{4}\right)
$$

which cannot be zero by the same arguments as before.

Thus we have checked by contradiction that $\left(m_{1}, \ldots, m_{5}\right)$ cannot be different from $(0, \ldots, 0)$.

Corollary 1. Under the assumptions of Proposition [3.4, let $X$ be an algebraic subvariety of $Z$ such that $X \cap\left(\mathbb{R}^{*}\right)^{5}$ is not empty.

Then the stabilizer group $\operatorname{Stab}(X)=\{g \in G: g \cdot X=X\}$ is discrete.

Proof. If $\operatorname{Stab}(X)$ is not discrete, it contains an algebraic subgroup $H$ isomorphic to $\mathbb{C}^{*}$, i.e., given as

$$
H=\left\{\left(z^{m_{1}}, \ldots, z^{m_{5}}\right): z \in \mathbb{C}^{*}\right\}
$$

with $\left(m_{1}, \ldots, m_{5}\right) \in \mathbb{Z}^{5} \backslash\{(0, \ldots, 0)\}$.

Since $X \cap\left(\mathbb{R}^{*}\right)^{5}$ is non-empty, there are $\alpha_{i} \in \mathbb{R}^{*}$ with $\left(\alpha_{1}, \ldots, \alpha_{5}\right) \in X$. Then

$$
\left(\alpha_{1} z^{m_{1}}, \ldots, \alpha_{5} z^{m_{5}}\right) \in X \forall z \in \mathbb{C}^{*}
$$

contradicting the preceding proposition.

Remark. The assumption that $X$ contains a real point is crucial. E.g., for $M=I_{4}$ consider

$$
X=\left\{(1,1, z, i z ; 2): z \in \mathbb{C}^{*}\right\} .
$$

Then $X \cap\left(\mathbb{R}^{*}\right)^{5}$ is empty and $\operatorname{Stab}(X)$ is 1-dimensional.

Theorem 3.5. Let $c_{1}, \ldots, c_{5} \in \mathbb{H}$ be given such that there is no proper real affine 3 -subspace of $\mathbb{H}$ containing all $c_{i}$.

Then every slice regular function $f: \mathbb{H} \rightarrow \mathbb{H}$ with $f(\mathbb{H}) \subset \mathbb{H} \backslash\left\{c_{1}, \ldots, c_{5}\right\}$ is constant.

Proof. Without loss of generality we may assume that $c_{5}=0$. By abuse of language we identify $c_{i} \in \mathbb{H}$ with $c_{i} \otimes 1 \in \mathbb{H}_{\mathbb{C}}$. Let $\langle$,$\rangle denote the complex bilinear form on$ $\mathbb{H}_{\mathbb{C}}$ induced by the euclidean scalar product on $\mathbb{H} \simeq \mathbb{R}^{4}$, i.e., $\langle z, w\rangle=\sum_{i} z_{i} w_{i}$. 
We define a holomorphic map $\phi: \mathbb{H}_{\mathbb{C}}=\mathbb{C}^{4} \rightarrow \mathbb{C}^{5}$ by

$$
\phi:\left(\begin{array}{c}
z_{1} \\
\vdots \\
z_{4}
\end{array}\right) \mapsto\left(\begin{array}{c}
\langle z, z\rangle-2\left\langle z, c_{1}\right\rangle+\left\langle c_{1}, c_{1}\right\rangle \\
\vdots \\
\langle z, z\rangle-2\left\langle z, c_{4}\right\rangle+\left\langle c_{4}, c_{4}\right\rangle \\
\langle z, z\rangle
\end{array}\right) .
$$

Observe that $\phi(z)=\overline{\phi(\bar{z})}$.

By assumption the vectors $c_{1}, \ldots, c_{4}$ form a real vector space basis for $\mathbb{H}$. It follows that there exists an invertible real $4 \times 4$-matrix $B$ such that

$$
\left(\begin{array}{c}
\left\langle z, c_{1}\right\rangle \\
\vdots \\
\left\langle z, c_{4}\right\rangle
\end{array}\right)=B^{-1} \cdot z \forall z \in \mathbb{R}^{4} \simeq \mathbb{H} .
$$

Let $M=B^{t} B$. Then $M$ is a positive definite symmetric real matrix $M$ such that for every $z \in \mathbb{C}^{4}$ we have

$$
\langle z, z\rangle=v^{t} \cdot M \cdot v
$$

if

$$
v=\left(\begin{array}{c}
\left\langle z, c_{1}\right\rangle \\
\vdots \\
\left\langle z, c_{4}\right\rangle
\end{array}\right)
$$

We observe that

$$
\phi_{i}(z)=\langle z, z\rangle-2\left\langle z, c_{i}\right\rangle+\left\langle c_{i}, c_{i}\right\rangle
$$

for $z=\left(z_{1}, \ldots, z_{4}\right)$ and $i \in\{1,2,3,4\}$ implies that

$$
\left\langle z, c_{i}\right\rangle=-\frac{1}{2}\left(\phi_{i}(z)-\langle z, z\rangle-\left\langle c_{i}, c_{i}\right\rangle\right) .
$$

Combined with $\phi_{5}(z)=\langle z, z\rangle$ we obtain that

$$
\phi_{5}(z)=v^{t} M v
$$

for

$$
v_{i}=-\frac{1}{2}\left(\phi_{i}(z)-\langle z, z\rangle-\left\langle c_{i}, c_{i}\right\rangle\right) .
$$

On $\mathbb{C}^{5}$ we define an algebraic subvariety $Z$ as the zero set of the function

$$
\begin{aligned}
& \psi\left(w_{1}, \ldots, w_{4} ; p\right)=p-u^{t} M u, \quad \text { with } \\
& u=-\frac{1}{2}\left(w_{1}-p-\left\langle c_{1}, c_{1}\right\rangle, \ldots, w_{4}-p-\left\langle c_{4}, c_{4}\right\rangle\right)^{t} .
\end{aligned}
$$

Due to the definition of $\psi$ it is clear that $\psi(w ; p)=0$ if $(w, p)=\phi(z)$ for some $z \in \mathbb{C}^{4}$.

Therefore $\phi\left(\mathbb{C}^{4}\right) \subset Z$.

We claim that $\phi: \mathbb{C}^{4} \rightarrow Z$ is biholomorphic. Indeed, consider

$$
\mu:\left(\begin{array}{c}
v_{1} \\
\vdots \\
v_{4} \\
v_{5}
\end{array}\right) \mapsto B \cdot\left(\begin{array}{c}
\left.-\frac{1}{2}\left(v_{1}-\left\langle c_{1}, c_{1}\right\rangle-v_{5}\right)\right) \\
\vdots \\
\left.-\frac{1}{2}\left(v_{4}-\left\langle c_{4}, c_{4}\right\rangle-v_{5}\right)\right)
\end{array}\right)
$$


with $B$ defined as in (3.2). Due to the definitions of $\phi$ and $B$ ((3.1), resp., (3.2) ) this map $\mu: Z \rightarrow \mathbb{C}^{4}$ is an inverse for $\phi: \mathbb{C}^{4} \rightarrow Z$. Thus $\mathbb{C}^{4}$ and $Z$ are biholomorphic and even isomorphic as algebraic varieties.

Now let $f$ be a non-constant slice regular function avoiding the values $c_{1}, \ldots, c_{4}$, $c_{5}=0$ and let $F: \mathbb{C} \rightarrow \mathbb{H}_{\mathbb{C}} \simeq \mathbb{C}^{4}$ be its stem function. Since $\phi\left(\mathbb{C}^{4}\right) \subset Z=\{\psi=0\}$, we obtain a holomorphic map $g=\phi \circ F: \mathbb{C} \rightarrow Z$. By construction $g(z)=\overline{g(\bar{z})}$ for all $z \in \mathbb{C}$. Furthermore $g$ is non-constant, because $F$ is non-constant and $\phi$ is injective.

Because $f: \mathbb{H} \rightarrow \mathbb{H}$ is assumed to avoid $c_{i}$ for every $i$, we know (thanks to Proposition 2.2) that $\phi_{i}(F(z)) \neq 0$ for all $z \in \mathbb{C}$ and all $i$, i.e., $\phi(F(\mathbb{C})) \subset Z \cap\left(\mathbb{C}^{*}\right)^{5}$.

Thus we may apply Proposition 3.3 and conclude that there exist $\alpha_{1} \ldots, \alpha_{5} \in \mathbb{R}^{*}$ and $\left(m_{1}, \ldots, m_{5}\right) \in \mathbb{Z}^{5} \backslash\{(0, \ldots, 0)\}$ such that $\zeta\left(\mathbb{C}^{*}\right) \subset Z$ for

$$
\zeta(z) \stackrel{\text { def }}{=}\left(\alpha_{1} z^{m_{1}}, \ldots, \alpha_{5} z^{m_{5}}\right) .
$$

But such a holomorphic map cannot exist due to Proposition 3.4. Contradiction! Thus there is no non-constant slice regular function $f: \mathbb{H} \rightarrow \mathbb{H}$ avoiding all the $c_{i}$.

Remark. If $f: \mathbb{H} \rightarrow \mathbb{H}$ is non-constant and slice preserving (i.e., it preserves each slice), then it can avoid only real points and at most one.

If $f$ is non-constant and one-slice preserving (i.e., it preserves a unique slice), then it can avoid only one point on the slice which is preserved.

\section{Big PICARD}

In complex analysis, the "Big Picard theorem" states the following: If $f$ is a holomorphic function on $\Delta^{*}=\{z \in \mathbb{C}: 0<|z|<1\}$ with an essential singularity at 0 , then $f$ assumes every value in $\mathbb{P}_{1}$ infinitely often with at most two exceptions.

Proposition 4.1. Let $Z$ be defined as in Proposition 3.4. Let $\eta$ be a holomorphic map from $\Delta^{*}$ to $Z \subset\left(\mathbb{C}^{*}\right)^{5} \subset\left(\mathbb{P}_{1}\right)^{5}$ with $\eta(\bar{z})=\overline{\eta(z)}$ for all $z$.

Then $\eta$ extends through 0 to a holomorphic map to $\left(\mathbb{P}_{1}\right)^{5}$, i.e., the isolated singularity of $\eta$ at 0 is not essential.

Proof. Let $X$ denote Zariski closure of $\eta\left(\Delta^{*}\right)$ in $Z$. Note that $\eta(z) \in\left(\mathbb{R}^{*}\right)^{5}$ for $z \in \mathbb{R} \cap \Delta^{*}$. Thus $X$ has non-trivial intersection with $\left(\mathbb{R}^{*}\right)^{5}$. It follows that $\operatorname{Stab}(X)$ is discrete (see Corollary 1 of Section 3). This implies that $\eta$ extends to a holomorphic map defined on $\Delta$ (Proposition 3.2).

Theorem 4.2 (Quaternionic Big Picard). Let $\mathbb{B}$ denote the open unit ball in $\mathbb{H}$ and let $f: \mathbb{B} \backslash\{0\} \rightarrow \mathbb{H}$ be a slice regular function with stem function $F: \Delta^{*} \rightarrow \mathbb{H}_{\mathbb{C}}$. Assume that $F$ has an essential singularity at 0 (i.e., at least one of the components of $F$ has an essential singularity).

Let $S$ denote the set of all $v \in \mathbb{H}$ for which the level set $f^{-1}(v)=\{q \in \mathbb{H}: f(q)=$ $v\}$ is finite.

Then $S$ is contained in an affine real hyperplane in $\mathbb{H}$.

Proof. Assume the contrary. Then there are five values $c_{0}, \ldots, c_{4}$ for which the level set is finite such that these five values generate $\mathbb{H}$ as an affine real space. Since 
$\bigcup_{m=0}^{4} f^{-1}\left(c_{m}\right)$ is finite, we may define

$$
r=\min \left\{|q|: q \in \bigcup_{m=0}^{4} f^{-1}\left(c_{m}\right), q \neq 0\right\}, \quad \mathbb{B}_{r}=\{q \in \mathbb{H}:|q|<r\} .
$$

Now $\left.f\right|_{\mathbb{B}_{r} \backslash\{0\}}$ avoids $c_{0}, \ldots, c_{4}$. Hence $\phi(F(z)) \in\left(\mathbb{C}^{*}\right)^{5} \cap Z$ for all $z \in \mathbb{C},|z|<r$ (with $\phi$ and $Z$ defined as in Theorem 3.5). Due to Proposition 4.1 the holomorphic map $\phi \circ F:\{z \in \mathbb{C}: 0<|z|<r\} \rightarrow Z$ extends to a holomorphic map with values in $\left(\mathbb{P}_{1}\right)^{5}$. But $\phi: \mathbb{H}_{\mathbb{C}} \rightarrow Z$ is a biholomorphic map, whose inverse map $\phi^{-1}=\mu$ is polynomial (see the proof of Theorem 3.5). It follows immediately that $\phi^{-1} \circ(\phi \circ F)=F$ extends to a holomorphic map from $\Delta$ to $\left(\mathbb{P}_{1}\right)^{4}$. This yields a contradiction to our assumptions.

Since over the complex field, Picard's theorems are the global version of the local Landau's Theorem, we point out that a quaternionic Landau's Theorem for slice regular functions already exists in the literature; see [3].

Proposition 4.3. For every non-constant slice regular function $f: \mathbb{H} \rightarrow \mathbb{H}$ the image is dense in $\mathbb{H}$.

Proof. If the image is not dense, its complement contains a non-empty open set. But it is trivially possible to choose five points in general position inside any given non-empty open set, leading to a contradiction with Theorem 3.5 .

In particular, a bounded slice regular function $f: \mathbb{H} \rightarrow \mathbb{H}$ must be constant, a fact which was first proved in [5, Theorem 3.7].

\section{The EXAmple of A FunCtion AVoiding $\mathbb{C}_{I}$}

Here we provide an example of a slice regular function avoiding infinitely many values.

Proposition 5.1. Let $f: \mathbb{H} \rightarrow \mathbb{H}$ be the slice regular function induced by the stem function

$$
F(z)=J \otimes \sin (z)+K \otimes \cos (z) .
$$

Then

$$
f(\mathbb{H})=\mathbb{H} \backslash \mathbb{C}_{I}=\left\{c_{1}+c_{2} I+c_{3} J+c_{4} K ; c_{i} \in \mathbb{R},\left(c_{3}, c_{4}\right) \neq(0,0)\right\} .
$$

Proof. We start with some preparations concerning complex trigonometric functions.

We recall that $\sin (i y)=i \sinh (y)$ and $\cos (i y)=\cosh (y)$ for all $y \in \mathbb{R}$.

For $z=x+i y(x, y \in \mathbb{R})$ we obtain

$$
\begin{aligned}
\sin (z)=\sin (x+i y) & =\sin (x) \cos (i y)+\cos (x) \sin (i y) \\
& =\sin (x) \cosh (y)+i \cos (x) \sinh (y)
\end{aligned}
$$

and

$$
\cos (z)=\cos (x+i y)=\cos (x) \cosh (y)-i \sin (x) \sinh (y) .
$$

Given $c=c_{1}+c_{2} I+c_{3} J+c_{4} K \in \mathbb{H}$, there exists a quaternionic number $q$ with $f(q)=c$ iff there exists a complex number $z=x+i y$ with

$$
\langle F(z)-c \otimes 1, F(z)-c \otimes 1\rangle=0 .
$$


Now

$$
\begin{aligned}
& \langle F(z)-c \otimes 1, F(z)-c \otimes 1\rangle \\
= & \langle F(z), F(z)\rangle-2\langle c \otimes 1, F(z)\rangle+\|c\|^{2} \\
= & 1-2\left(c_{3} \sin (z)+c_{4} \cos (z)\right)+\|c\|^{2}
\end{aligned}
$$

implying

$$
\Im(\langle F(z)-c \otimes 1, F(z)-c \otimes 1\rangle)=-2 \sinh (y)\left(c_{3} \cos (x)-c_{4} \sin (x)\right)
$$

and

$$
\begin{aligned}
\Re(\langle F(z)-c \otimes 1, F(z)-c \otimes & 1\rangle) \\
& =1-2 \cosh (y)\left(c_{3} \sin (x)+c_{4} \cos (x)\right)+\|c\|^{2} .
\end{aligned}
$$

It follows that

$$
\Re(\langle F(z)-c \otimes 1, F(z)-c \otimes 1\rangle)=1+\|c\|^{2} \geq 1>0
$$

if $c_{3}=c_{4}=0$. This proves that $f$ does not assume any value in $\mathbb{C}_{I}$.

It remains to prove that all other values are assumed.

We claim: For every $c \in \mathbb{H} \simeq \mathbb{R}^{4}$ with $\left(c_{3}, c_{4}\right) \neq(0,0)$ there exist $x, y \in \mathbb{R}$ such that $\langle F(x+y i)-c \otimes 1, F(x+y i)-c \otimes 1\rangle=0$.

First we choose $x \in \mathbb{R}$ such that

$$
c_{3} \cos (x)-c_{4} \sin (x)=0 .
$$

Due to (5.1) this guarantees that

$$
\Im(\langle F(x+y i)-c \otimes 1, F(x+y i)-c \otimes 1\rangle)=0 .
$$

If $c_{3} \sin (x)+c_{4} \cos (x)<0$, we replace $x$ by $x+\pi$. This ensures that

$$
c_{3} \sin (x)+c_{4} \cos (x)>0 .
$$

Define

$$
t=\frac{1+\|c\|^{2}}{2\left(c_{3} \sin x+c_{4} \cos x\right)} .
$$

We have to show that there exists a number $y \in \mathbb{R}$ with $\cosh (y)=t$, because then it follows from (5.1) and (5.2) that $\langle F(x+i y), F(x+i y)\rangle=0$.

An application of the Cauchy Schwarz Inequality to the vectors $\left(c_{3}, c_{4}\right)$ and $(\sin (x), \cos (x))$ yields the inequality

$$
\left|c_{3} \sin (x)+c_{4} \cos (x)\right| \leq \sqrt{c_{3}^{2}+c_{4}^{2}} .
$$

Using $c_{3} \sin (x)+c_{4} \cos (x)>0$ it follows that

$$
t=\frac{1+\|c\|^{2}}{2\left(c_{3} \sin x+c_{4} \cos x\right)} \geq \frac{1+\left(c_{3} \sin x+c_{4} \cos x\right)^{2}}{2\left(c_{3} \sin x+c_{4} \cos x\right)} \geq 1 .
$$

Now $t \geq 1$ implies that there exists a real number $y$ with $\cosh (y)=t$. This completes the proof. 


\section{Avoiding three points}

Proposition 6.1. Let $c_{1}, c_{2}, c_{3}$ be three arbitrary quaternionic numbers.

Then there exists a non-constant slice regular function $f(q)=\sum q^{k} a_{k}$ such that $f(\mathbb{H}) \subset \mathbb{H} \backslash\left\{c_{1}, c_{2}, c_{3}\right\}$.

Proof. We have seen that there exists a slice regular function $f(q)=\sum_{k} q^{k} a_{k}$ with $f(\mathbb{H}) \subset \mathbb{H} \backslash \mathbb{C}_{I}$ (Proposition 5.1).

We modify this function in the following way: Let $\lambda \in \mathbb{H}^{*}, p \in \mathbb{H}$ and let $\phi$ be a ring automorphism of $\mathbb{H}$.

Then we define a slice regular function $g$ by

$$
g(q) \stackrel{\text { def }}{=}\left(\sum_{k} q^{k} \phi\left(a_{k}\right)\right) \lambda+p .
$$

For any $c \in \mathbb{H}$ we have

$$
\begin{aligned}
c & =g(\phi(q)) \\
\Longleftrightarrow c & =\phi(f(q)) \lambda+p \\
\Longleftrightarrow \phi^{-1}(c) & =f(q) \phi^{-1}(\lambda)+\phi^{-1}(p) \\
\Longleftrightarrow f(q) & =\left(\phi^{-1}(c)-\phi^{-1}(p)\right) \phi^{-1}(1 / \lambda) .
\end{aligned}
$$

Let $c_{1}, c_{2}, c_{3} \in \mathbb{H}$ be three given distinct quaternionic numbers. (Evidently it suffices to consider only the case of three distinct numbers.)

We choose $p, \lambda, \phi$ such that:

(i) $p=c_{1}$,

(ii) $\lambda=c_{2}-c_{1}$,

(iii) $\phi^{-1}\left(\left(c_{3}-c_{1}\right)\left(c_{2}-c_{1}\right)^{-1}\right) \in \mathbb{C}_{I}$.

In order to verify that this is possible, let $H \in \mathbb{H}$ be an imaginary unit (i.e., $H^{2}=$ $-1)$ such that

$$
\left(c_{3}-c_{1}\right)\left(c_{2}-c_{1}\right)^{-1} \in \mathbb{C}_{H}=\mathbb{R} \oplus H \mathbb{R} .
$$

Let $\phi$ be an orientation preserving linear orthogonal transformation of $\mathbb{H}$ fixing $\mathbb{R}$ pointwise and such that $\phi(I)=H$. Then $\phi$ is a ring automorphism of $\mathbb{H}$ satisfying (iii).

It is easily verified that

$$
\left(\phi^{-1}\left(c_{i}\right)-\phi^{-1}(p)\right) \phi^{-1}(1 / \lambda) \in \mathbb{C}_{I}
$$

for all three indices $i \in\{1,2,3\}$. Since $f$ avoids values in $\mathbb{C}_{I}$, it follows that $g$ avoids the three values $c_{1}, c_{2}, c_{3}$.

Remark. Since any 2-dimensional real affine subspace $P$ of $H \simeq \mathbb{R}^{4}$ is spanned by three points, it follows from the above that there exists an entire slice regular function $f: \mathbb{H} \rightarrow \mathbb{H}$ such that $f(\mathbb{H})=\mathbb{H} \backslash P$.

Open Problem. Is or isn't there a non-constant slice regular entire function of $\mathbb{H}$ avoiding four general points?

\section{OCTONIONS}

In view of the results of [6], in particular theorem 17 , one may easily modify our arguments in order to obtain a Picard theorem for the algebra of octonions, namely we have the following. 
Theorem 7.1. For every non-constant slice regular function $f: \mathbb{O} \rightarrow \mathbb{O}$ the set $\mathbb{O} \backslash f(\mathbb{O})$ is contained in a real affine hyperplane of $\mathbb{O}$.

\section{REFERENCES}

[1] D. Angella and C. Bisi, Slice-quaternionic Hopf surfaces, J. Geom. Anal. 29 (2019), no. 3, 1837-1858, DOI 10.1007/s12220-018-0064-9. MR.3969415

[2] C. Bisi and G. Gentili, On quaternionic tori and their moduli space, J. Noncommut. Geom. 12 (2018), no. 2, 473-510, DOI 10.4171/JNCG/284. MR.3825194

[3] C. Bisi and C. Stoppato, Landau's theorem for slice regular functions on the quaternionic unit ball, Internat. J. Math. 28 (2017), no. 3, 1750017, 21, DOI 10.1142/S0129167X17500173. MR.3629144

[4] G. Gentili, C. Stoppato, and D. C. Struppa, Regular functions of a quaternionic variable, Springer Monographs in Mathematics, Springer, Heidelberg, 2013. MR.3013643

[5] G. Gentili and D. C. Struppa, A new theory of regular functions of a quaternionic variable, Adv. Math. 216 (2007), no. 1, 279-301, DOI 10.1016/j.aim.2007.05.010. MR2353257

[6] R. Ghiloni and A. Perotti, Slice regular functions on real alternative algebras, Adv. Math. 226 (2011), no. 2, 1662-1691, DOI 10.1016/j.aim.2010.08.015. MR2737796

[7] S. Mongodi, Holomorphicity of slice-regular functions, Complex Anal. Oper. Theory 14 (2020), no. 3, Paper No. 37, 26, DOI 10.1007/s11785-020-00996-2. MR4078414

[8] J. Noguchi, On holomorphic curves in semi-abelian varieties, Math. Z. 228 (1998), no. 4, 713-721, DOI 10.1007/PL00004640. MR1644444

[9] J. Noguchi, Lemma on logarithmic derivatives and holomorphic curves in algebraic varieties, Nagoya Math. J. 83 (1981), 213-233. MR632655

[10] E. Picard, Sur une propriété des fonctions entières, C. R. Acad. Sci. Paris 88 (1879), 10241027.

[11] E. Picard, Sur les fonctions entières, C. R. Acad. Sci. Paris 89 (1879), 662-665.

[12] É. Picard, Mémoire sur les fonctions entières (French), Ann. Sci. École Norm. Sup. (2) 9 (1880), 145-166. MR1508689

[13] S. J. Sangwine and D. Alfsmann, Determination of the biquaternion divisors of zero, including the idempotents and nilpotents, Adv. Appl. Clifford Algebr. 20 (2010), no. 2, 401-410, DOI 10.1007/s00006-010-0202-3. MR2645358

Department of Mathematics and Computer Sciences, Ferrara University, Via MachiAVELli 30, 44121 FERRARA, ItAly

Email address: bsicnz@unife.it

IB 3/111, Lehrstuhl Analysis II, Fakultät für Mathematik, Ruhr-Universität Bochum, 44780 Bochum, Germany

Email address: joerg.winkelmann@rub.de 